\title{
Monitoring Techniques for the Manufacture of Tapered Optical Fibres
}

\author{
Kevin Mullaney, Ricardo Correia, Stephen E. Staines, Stephen W. James* \\ AND RALPH P. TATAM \\ Engineering Photonics, School of Engineering, Cranfield University, Bedford, MK43 OAL, UK.
}

*Corresponding author: s.w.james@cranfield.ac.uk

Received XX Month XXXX; revised XX Month, XXXX; accepted XX Month XXXX; posted XX Month XXXX (Doc. ID XXXXX); published XX Month XXXX

The use of a range of optical techniques to monitor the process of fabricating optical fibre tapers is investigated. Thermal imaging was used to optimize the alignment of the optical system: the transmission spectrum of the fibre was monitored to confirm that the tapers had the required optical properties and the strain induced in the fibre during tapering was monitored using in-line optical fibre Bragg gratings. Tapers were fabricated with diameters down to $5 \mu \mathrm{m}$ and with waist lengths of $20 \mathrm{~mm}$ using single-mode SMF-28 fibre.

OCIS codes: (060.2370) Fibre optic sensors; (060.3735) Fibre Bragg gratings; (280.4788) Optical sensing and sensors; (350.3390) Laser materials processing.

http://dx.doi.org/10.1364/AO.99.099999

\section{Introduction}

Tapered optical fibres have applications in telecommunications as directional couplers [1] and can also be used as sensing devices for a wide range of bio-medical and chemical applications [2 - 4]. A tapered fibre is fabricated by locally heating a short section of optical fibre while stretching it. This results in a length of fibre that has a narrow waist. In the region of the taper waist, the evanescent field of the propagating mode extends into the medium around the fibre, so allowing the interaction of light with the surrounding medium. The light transmitted by a tapered fibre is sensitive to changes in the refractive index of the medium within the vicinity of the taper waist and this feature forms the basis for the use of tapers as interferometric refractometers $[5,6]$. Tapered optical fibres have been used for a variety of sensing applications: they have been used to monitor in realtime the growth of Escherichia coli in an aqueous medium [2], for the detection of label free single stranded DNA in real time [7], for Raman spectroscopic sensing [8] and as gas sensors [4]. Tapers have also been used to couple light to a microsphere so allowing wavelength selective fibre lasers [9] to be made for environmental sensing applications.

The heat source employed during taper fabrication is usually either a flame which is stationary or oscillating [10 - 13], a CO2 laser [14 - 16], micro-heaters in the form of electric strip heaters [17] and sapphire tubes [18]. The sapphire tube is heated using a $\mathrm{CO}_{2}$ laser.

The use of a $\mathrm{CO}_{2}$ laser has a number of advantages over the use of a flame. The heated-zone properties can be controlled in a repeatable fashion and the use of a laser avoids the production of air turbulence and combustion contaminants, present when using a flame, which potentially can influence the shape, uniformity and cleanliness of the tapered region [16].
Using $\mathrm{CO}_{2}$ lasers, tapers have been fabricated with waist diameters down to $4 \mu \mathrm{m}[14,15,16,19]$ and with waist lengths of up to $20 \mathrm{~mm}$. The tapering processes that were developed by these researchers were focused primarily at fabricating low loss tapers for use as fibre couplers and so emphasis was placed on producing tapers with insertion losses of less than $0.4 \mathrm{~dB}$. Using a sapphire tube heated by a $\mathrm{CO}_{2}$ laser, has produced waist diameters of $700 \mathrm{~nm}$ [18], creating a nanofibre. High transmission nanofibres with diameters less than 600 $\mathrm{nm}$ have also been fabricated using oscillating flames [13]. These low loss tapers have been used for coupling light to micro-resonators.

Tapered optical fibres used in bio-chemical sensing applications often have diameters in the range 5 to $20 \mu \mathrm{m}$ [2 - 4], and have nonadiabatic taper profiles to allow the use of modal interferometry to probe changes in the refractive index of the medium or of the properties of a functional coating. The index sensitivity of the device increases as the taper waist diameter decreases [4], however the visibility of the channelled transmission spectrum reduces as the waist diameter reduces to $\sim 1 \mu \mathrm{m}$ [13]. These taper waist diameters are readily achievable using a $\mathrm{CO}_{2}$ laser as the heat source. The optical properties of these tapers are highly sensitive to the taper's physical geometry and so the ability to make reproducible tapers is important. To understand and optimize the process for sensing applications, the use of in-situ process monitoring techniques would be beneficial

There have been limited reports in the literature of systems to monitor the tapering process. Insertion loss was measured $[15,19]$ during the tapering process by coupling the output from a laser diode into the fibre and monitoring the transmitted power. The force experienced by the fibre during the tapering process was monitored using a load cell [19]. The data produced by the load cell was used to provide information to the operator so the laser power could be 
adjusted manually during the process with the aim of maintaining the fibre at the melting temperature. The way in which the load cell was incorporated into the system caused the fibre to move during the stretching process. As this fibre movement was perpendicular to the fibre axis, the shape of the taper was influenced, resulting in large variations in insertion loss between the fabricated tapers. Aharoni et al. [20] monitored successfully the temperature of tapered fibre preforms during the drawing process using a long wave infra-red camera.

The aim of the paper is to explore techniques that can be used to monitor process parameters of importance to the fabrication of tapers. The monitoring approaches are: thermal imaging of the heated fibre using a near infra-red (NIR) camera, monitoring the visible and NIR spectrum of the light transmitted though the taper using a spectrophotometer and the use of an in-line fibre Bragg grating (FBG) as a strain sensor. $\mathrm{A} \mathrm{CO}_{2}$ laser is used as the heat source, which limits the minimum taper waist diameter achievable, but which is adequate for many applications. The observations on the value of the use of the monitoring techniques would be applicable to taper fabrication approaches using other heat sources.

\section{Fibre taper fabrication system}

The optical fibre taper fabrication system employed is shown in figure 1, together with the co-ordinate definition. The output from a Synrad 48-2 $\mathrm{CO}_{2}$ laser, with a maximum output power of $25 \mathrm{~W}$, was used to heat the fibre. ZnSe optical elements were used for power tapping and injecting the HeNe alignment laser into the path of the $\mathrm{CO}_{2}$ laser beam. The galvanometer mounted mirror (Cambridge Technology 6210) was driven with a $100 \mathrm{~Hz}$ triangular waveform to provide uniform illumination along the section of fibre to be tapered. A triangular waveform was found to give improved uniformity compared to a sinusoidal driving waveform as it provided a constant mirror velocity over the driving cycle. The $100 \mathrm{~Hz}$ frequency was found to be the optimum trade-off between mirror motion linearity, which decreased with increasing frequency, and mirror speed, as the sweep of the laser beam along the fibre was fast enough to ensure the fibre did not cool between successive passes of the beam. A ZnSe planoconvex cylindrical lens with a $100 \mathrm{~mm}$ focal length, in conjunction with progressively increasing laser power, was used to achieve the required energy flux at the fibre, so allowing the softening temperature to be maintained as the fibre diameter decreased [18]. The laser flux (2.6 $11 \mathrm{~kW} / \mathrm{cm}^{2}$ ) required to soften the fibre was determined experimentally by observing the heated fibre using a NIR camera (Vosskuhler NIR-300P) and noting the laser power that resulted in the fibre "bowing". The "bowing" occurs near the fibre softening temperature, which has been shown to be $\sim 910{ }^{\circ} \mathrm{C}$ [14]. The focal length of the $\mathrm{ZnSe}$ cylindrical lens was chosen to achieve the required laser flux density at the fibre and, together with the beam waist and Rayleigh range, provided acceptable tolerances on the alignment requirements in the $\mathrm{x}, \mathrm{y}$ and $\mathrm{z}$ planes (figure 1 ).

The beam illuminating the fibre had a scanned length of $8 \pm 0.25$ $\mathrm{mm}$ and a width in the y-plane of $355 \pm 30 \mu \mathrm{m}$. These values were determined by melting the frosted surface of acrylic test-pieces with the laser operating at $4 \mathrm{~W}$. The acrylic used was Plexiglas GS supplied by Rohm. The use of lenses of shorter focal lengths were found to impact on the tolerances required in the mechanical aspects of the fabrication system. For example, a smaller depth of focus would result in a significant variation in the laser flux density at the fibre during the tapering process as the fibre can bow in the z-direction, particularly if the rate at which the fibre is pulled is slower than the rate of increase of length due to thermal expansion. In the y-direction, the use of a shorter focal length lens reduces the beam width, reducing the tolerance on the location of the central axis of the fibre relative to the centre of the beam along its minor elliptical axis.

Two programmable rotation stages (Physik Instrumente) supported and located the fibre to be heated and stretched. The capstans mounted on the stages were inscribed with v-grooves to ensure reproducible fibre placement in the focal plane of the $\mathrm{ZnSe}$ lens. The stages started pulling the fibre when the temperature of the fibre was at the softening point, determined by observation of bowing of the fibre, as described previously. The operation of the laser, galvanometer and rotation stages were controlled using a program written in Labview, which provided the requisite driving waveforms to control the laser power and the movement of the rotation stages. In addition, the program provided the necessary synchronization between the process steps, such that the process was repeatable.

To optimize and to ensure repeatability of the process, three monitoring techniques were deployed. An NIR camera was used to aid the alignment of the heated fibre within the focused laser spot and to observe both the thermal intensity distribution along the fibre [14] and displacement of the fibre ("bowing") during the pulling process. A CCD spectrophotometer and a tungsten-halogen light source were used to facilitate the measurement of the transmission spectrum of the optical fibre over the wavelength range 500-1150 nm, which allowed the tapering process to be terminated when the desired transmission spectrum was achieved (option A in figure 1). Finally, the strain experienced by the fibre was monitored during the heat and stretch process using an FBG (option B in figure 1).

\section{Experimental Results and Discussion}

Observation of the temperature distribution along the fibre using an NIR camera allows the impact of the alignment of the laser on the taper geometry to be assessed. To achieve uniform heating along the section of optical fibre to be tapered, it was found that the narrow Gaussian spot formed by the lens should be located along the central axis of the fibre as it was swept longitudinally along the fibre axis by the galvanometer mirror. In addition, the alignment of the laser beam with respect to the rotation axis of the galvanometer mirror was also found to be particularly important when producing symmetrical tapers.

As discussed earlier, the decrease in diameter of the fibre during the tapering process requires an increase in the laser power to maintain the fibre at the melting temperature. Previous researchers [14 - 16], have observed visually the incandescent light emitted by the heated fibre and adjusted the laser power to produce a constant light level by eye. In this work, to determine the laser power profile required to maintain the fibre at the melting point over the duration of the tapering process, the heated area was imaged by the IR camera and the increasing power level which minimized the fibre deviation in the zdirection was recorded. The spectral response of the IR camera covers the wavelength range $900-1700 \mathrm{~nm}$ and thus is sensitive to the blackbody thermal emission spectra from the heated fibre. The camera spectral response and calculated Planck radiated power curves [21] for a range of temperatures near the fibre melting point of $1183 \mathrm{~K}$ are given in figure 2 . The camera becomes sensitive to the blackbody radiation emitted by the heated fibre once the fibre temperature exceeds $\sim 773 \mathrm{~K}$ and then with increasing sensitivity up to and beyond the melting point of the fibre. It was not possible to measure actual fibre temperature at this stage of the work as this requires accurate data on the fibre emissivity, which itself is a function of temperature [21]. In addition, because the fibre diameter, and hence radiant area, is reducing during the tapering process, this will complicate further the fibre temperature measurement. In the future, the capability of measuring fibre temperature will be explored as it allows the possibility of controlling the laser power more accurately using feedback control techniques.

A typical taper profile produced by this system under optimised alignment and power conditions is shown in figure 3 . The fibre diameter was measured using an Olympus BX51 microscope at $2 \mathrm{~mm}$ intervals along the tapered fibre. The taper was $34 \pm 0.2 \mathrm{~mm}$ long and had an average waist diameter of $5.0 \pm 0.5 \mu \mathrm{m}$. The transition zones were $11 \mathrm{~mm}$ long. The taper angle was 5.63 mrads and the insertion loss was $1.07 \mathrm{~dB}$. During tapering, the laser beam was scanned over a length of $8 \mathrm{~mm}$ and the power was increased from $3.8 \mathrm{~W}$ to $16 \mathrm{~W}$ as 
the fibre diameter reduced. During the time interval from 0 - 102 seconds the laser power is increased slowly to preheat the fibre. From 102 seconds the stages are activated and start stretching the fibre. The progressive power increase maintains the fibre at the softening temperature as the fibre diameter reduces.

The taper diameter profile can be predicted theoretically, using the "constant hot-zone model", described by Birks et al. [22]. It is based on the assumption that the volume of the heated fibre is conserved during the heating process. The model also assumes that a fixed length cylindrical section of fibre is heated to a uniform temperature and stretched symmetrically by pulling the fibre with equal and opposite constant velocities. Outside the heated region the fibre is assumed to be cold and solid. Birks et al. have shown that the taper radius profile has a decaying exponential profile (1) with the following function:

$$
r(z)=r_{0} \exp (-z / L)
$$

Where $r$ is the taper radius, $r_{0}$ is the initial fibre radius, $\mathrm{z}$ is the pull length and $L$ is the length of the hot-zone. Knowing the initial fibre radius and the taper diameter profile, L was varied until good agreement was achieved with the experimentally measured profile. Figure 3 shows the profile predicted by this model, assuming a hotzone length of $4.5 \mathrm{~mm}$. The hot-zone length is less than the scan length ( $8 \mathrm{~mm})$ as only the central section of the scanned length exceeds the softening temperature of the fibre.

Figure 4 shows the profile of taper $B$ fabricated using a lower power than taper A, and with an estimated hot-zone length of $2.5 \mathrm{~mm}$. The taper angle is 12.4 mrads with an associated insertion loss of $2.5 \mathrm{~dB}$. Taper B is $11 \mathrm{~mm}$ long with an average waist diameter of $14.0 \pm 0.5$ $\mu \mathrm{m}$. The transition zones were $\sim 5 \mathrm{~mm}$ long. Again, the taper profile, shown in figure 4 shows close agreement with the theoretical prediction. Ten of these tapers were made with a typical waist variation of $\pm 0.5 \mu \mathrm{m}$, indicating that the process was repeatable.

Figure 5 shows the evolution of the channelled transmission spectrum that is characteristic of tapers of diameter of order $10 \mu \mathrm{m}$ [3]. In the region of the taper waist, light propagates at the air/cladding interface, as here the single-mode fibre converts to multi-mode fibre behaviour. The interference between the two (or more) modes of the taper at the second transition zone results in a characteristic channelled transmission spectrum, a manifestation of the different effective refractive indices of the modes in the tapered region.

As the phase and period of the channelled spectrum changes in response to the reduction of the fibre diameter, the intensity measured at a fixed wavelength is modulated, as shown in figure 6 for a wavelength of $800 \mathrm{~nm}$. At $102 \mathrm{~s}$, the rotation stages were activated and start to stretch the heated fibre. Periodic fluctuations in the light transmitted by the fibre at a wavelength of $800 \mathrm{~nm}$ are first observed at $\sim 150 \mathrm{~s}$ when the waist diameter is less than $25 \mu \mathrm{m}$. As the taper angle for taper B is larger than in taper A, more light is coupled into the higher order modes [23] and hence the oscillation amplitude is greater than in taper $\mathrm{A}$.

As discussed earlier, Grellier [19] used a load cell to determine the strain experienced by the fibre during the tapering process. The disadvantage of this technique was that the fibre was perturbed from the focal plane of the laser as the fibre tension varied. In this research, we overcame this difficulty by using an FBG as an in-line intrinsic strain sensor. To measure the strain experienced by the fibre during the fabrication process, the FBG was located $12 \mathrm{~mm}$ from the heating zone to ensure that the strain measurements were not compromised by either heat conducted along the fibre or laser radiation scattered by the fibre. This distance was established by a series of experiments in which the fibre was heated to just below its melting point and the position of the heating zone relative to the FBG was varied while measuring the wavelength of the FBG reflection peak. The separation distance of the heated zone and the FBG where the reflection peak did not change was noted. In these experiments, the FBG was unstrained so that any displacement in the FBG spectrum was due to thermal conduction along the fibre.

The FBGs used in these experiments were fabricated in-house using hydrogen loaded single mode fibre (SMF-28). They were written by UV exposure through a phase mask using an injection-seeded frequencyquadrupled Nd:YAG laser operating at a wavelength of $266 \mathrm{~nm}$. Each FBG had a reflectivity of $3 \mathrm{~dB}$ and a length of $6 \mathrm{~mm}$. A SmartScan interrogator (Smartfibres Ltd) was used to monitor the Bragg wavelength, and thus the strain experienced by the fibre, while it was being pulled during the tapering process.

The strain measured by the FBG during taper fabrication is shown in figure 7, together with the measured and programmed variation in $\mathrm{CO}_{2}$ laser power. The power fluctuations were caused by mode-hopping. At $102 \mathrm{~s}$, the fibre was stretched, resulting in an abrupt increase in the fibre strain. As the laser power increases, the fibre starts to soften, resulting in a subsequent decrease in strain. As the fibre was then heated and pulled, no significant change in the strain was observed until 220 s elapsed. At this time, the strain increases abruptly, as the laser intensity incident on the reduced-diameter fibre was insufficient to maintain the fibre at the softening temperature and so the fibre solidifies [19]. Just before this occurred (at $215 \mathrm{~s}$ ), the laser power fluctuations and the measured strain varied in sympathy as the temperature of the fibre decreased and increased. At $224 \mathrm{~s}$, the fibre broke, with a correspondingly abrupt reduction in strain. In this experiment, the laser had not been pre-heated prior to tapering the fibre. This caused the power instability observed from $175 \mathrm{~s}$ onwards. A separate experiment was undertaken with the laser pre-heated by operating it an output power of $10 \mathrm{~W}$ for 25 minutes before the tapering process started. The results are shown in figure 8 . The laser pre-heating caused the laser output power to be more stable, which reduced the variation in the strain measured by the FBG. Bragg gratings can be used as a sensitive indicator of the strain experienced by the fibre during the tapering process and they offer the potential to optimize the control of the rotation stages, the laser power profile and in determining the onset of fibre breakage when fabricating tapers with small diameters. .

We suggest that the FBGs would be used to provide data of value for developing and optimizing the tapering process parameters, rather than for routine taper fabrication as the requirement for an FBG to be incorporated in the fabrication of each taper would increase the fibre handling time and could reduce the mechanical integrity of the tapered fibre.

\section{Conclusions}

During the course of this work, a variety of different tapers were fabricated. These tapers had waist diameters down to $5 \mu \mathrm{m}$ and had waist lengths of $\sim 20 \mathrm{~mm}$ using single-mode SMF-28 fibre. A range of optical techniques to monitor the process of fabricating optical fibre tapers has also been investigated. A camera operating in the near infrared has been used both to aid fibre alignment and to allow the laser power profile for the process to be easily determined.

Monitoring the transmission spectrum of the fibre during the tapering process allows the operator to ensure that the taper will have the required spectral properties in the wavelength region of interest. The use of an IR camera, together with monitoring the transmission of the fibre, are effective process tools so allowing the taper process to be optimized for specific sensing applications.

FBGs have been used to measure strain during the stretching process and can be used to precisely time the onset of fibre pulling, optimizing the $\mathrm{CO}_{2}$ laser power profile and identifying when fibre breakage is imminent. In this context, FBGs can be used for developing and optimizing the tapering process but not for routine taper production, as using FBGs increases fibre handling time and could reduce the mechanical integrity of the tapered fibre.

Future work will explore the effectiveness of using the IR camera to measure fibre temperature during the process so allowing the laser 
power to be actively controlled, thereby eliminating the need for unique laser power curves for tapers of differing geometries. The taper transitions which have been explored in this paper have been exponentially shaped, future work will also look at linear, convex and concave transition zones and their consequent impact on the optical performance of tapers.

Acknowledgements. We would like to thank the Engineering and Physical Sciences Research Council (EPSRC), UK for supporting this work via grant $\mathrm{EP} / \mathrm{H} 02252 \mathrm{X}$. For inquiries relating to access to the research data or other materials referred to in this article, please contact Cranfield University Library and Information Services, reseachdata@cranfield.ac.uk.

\section{References}

1. C. McAtamney, A. Cronin, R. Sherlock, G.M. O`Connor and T.J. Glynn, "Reproducible method for fabricating fused biconical tapered couplers using a $\mathrm{CO}_{2}$ laser based process", Proceedings of the third international WLT-Conference on lasers in manufacturing, (2005).

2. M. Zibaii, A. Kazemi, H. Latifi, M. Azar, S. Hosseini and M. Ghezelaiagh, "Measuring bacterial growth by refractive index tapered optical fibre biosensor," J. Photochemistry and Photobiology B: Biology 101, 313320 (2010).

3. R. Jarzebinska, C. Cheung, S. W. James and R. P. Tatam, "Response of the transmission spectrum of tapered optical fibres to the deposition of a nanostructured coating," Meas. Sci. Technol. 20, 034001, (2009).

4. R. Jarzebinska, S. Korposh, S. W. James, W. J. Batty, R. P, Tatam and S-W. Lee, "Optical gas sensor fabrication based on porphyrin-anchored electrostatic self-assembly onto tapered optical fibres," Anal. Lett. 45, 1297-1309 (2012).

5. S. Lacroix, F. Gonthier, R. Black and J. Bures, "Tapered-fibre interferometric wavelength response- the achromatic fringe," Opt. Lett. 13, 395-397, (1988).

6. F. Gonthier, J. Lapierre, C. Veilleux, S. Lacroix, and J. Bures, "Investigation of power oscillations along tapered monomode fibres," Appl. Opt. 26, 444-449 (1987).

7. M. Zibaii, H. Latifi, E. Ghanati, M. Gholami and S. Hosseini, "Label free detection of DNA hybridization by refractive index tapered fiber biosensor, Proc. SPIE, 7715, 1-9 (2010).

8. P. Edwards, C. Janisch, B. Peng, J. Zhu, S. Ozdemir, L. Yang and Z. Liu, "Label-Free Particle Sensing by Fiber Taper-Based Raman Spectroscopy, IEEE Photonics Techn. Lett., 26, 2093-2096 (2014).
9. S. Chen, T. Yeo, W. Zhao, T. Sun, K. Grattan, R. Lade, B. Powell. G. FosterTurner and M. Osbourne, "Development of a multi-wavelength microsphere fibre laser system for potential sensor applications, Optics Comm., 282, 401-405 (2009).

10. T. Allsop, F. Floreani, K. Jedrzejewski, P. Marques, R. Romero, D. J. Webb and I. Bennion, "Refractive index sensing with long period grating fabricated in biconical tapered fibre," Electron. Lett. 41, 471-472 (2005).

11. J. Villatoro, D. Monzon-Hernandez and E. Mejia, "Fabrication and modelling of uniform-waist single-mode tapered optical fibre sensors," App. Opt. 42, 2278-2283 (2003).

12. J.M. Ward, A. Maimaiti, V. H. Le and S. Chromaic, "Contributed Review: Optical micro- and nanofiber pulling rig", Rev. Sci. Inst. 85, 111501, (2014).

13. R. Nagai and T. Aoki, "Ulta-low-loss tapered optical fibers with minimal lengths",Opt. Expr. 22, No. 23, (2014).

14. F. Bayle and J. Meunier, "Efficient fabrication of a fused -fibre biconical taper structures by a scanned $\mathrm{CO}_{2}$ laser beam technique," Appl. Opt. 44, 6402-6411, (2005).

15. J. Ward, D. O'Shea, B. Shortt, M. Morrissey, K. Deasy and S. Chormaic, "Heat-and-pull rig for taper fabrication", Rev. Sci. Inst. 77, 083105 (2006).

16. T. Dimmick, G. Kakarantzas, T. Birks and P. St. J. Russell, "Carbon dioxide laser fabrication of fused-fibre couplers and tapers," Appl. Opt. 38, 6845-6848 (1999).

17. Y. Takeuchi and M. Horiguchi, "Microheater control of wavelengthflattened fiber coupler properties", App. Optics, 33, No. 6, (1994).

18. M. Sumetsky, Y. Dulashko and A. Hale, "Fabrication and study of bent and coiled free silica nanowires: Self-coupling microloop optical interferometer", Optics Express, 12, 3521-3531 (2004).

19. A. Grellier, "Characterisation of optical fibre tapers using a $\mathrm{CO}_{2}$ laser", PhD Thesis, University of Canterbury, UK, (2000).

20. R. Aharoni, L. Bidani, M. Sinvani and Z. Zalevsky, "Initiatory concept of localized laser-based tapering rig for realization of in-fiber devices", Opt. Eng., 51(7), 1-8, (2012).

21. W. Rees, "Physical Principles of Remote Sensing", Cambridge University Press, (2001).

22. T. Birks and Y. Li, “The shape of fibre tapers," J. of Lightwave Technol. 10, 432-438 (1992).

23. G. Cohoon, C. Boyter, M. Errico, K. Vandervort and E. Salik, "Enhancing sensitivity of biconical tapered fiber sensors with multiple passes through the taper," Opt. Eng. 49(3), 034401 (2010).

\section{Figures}

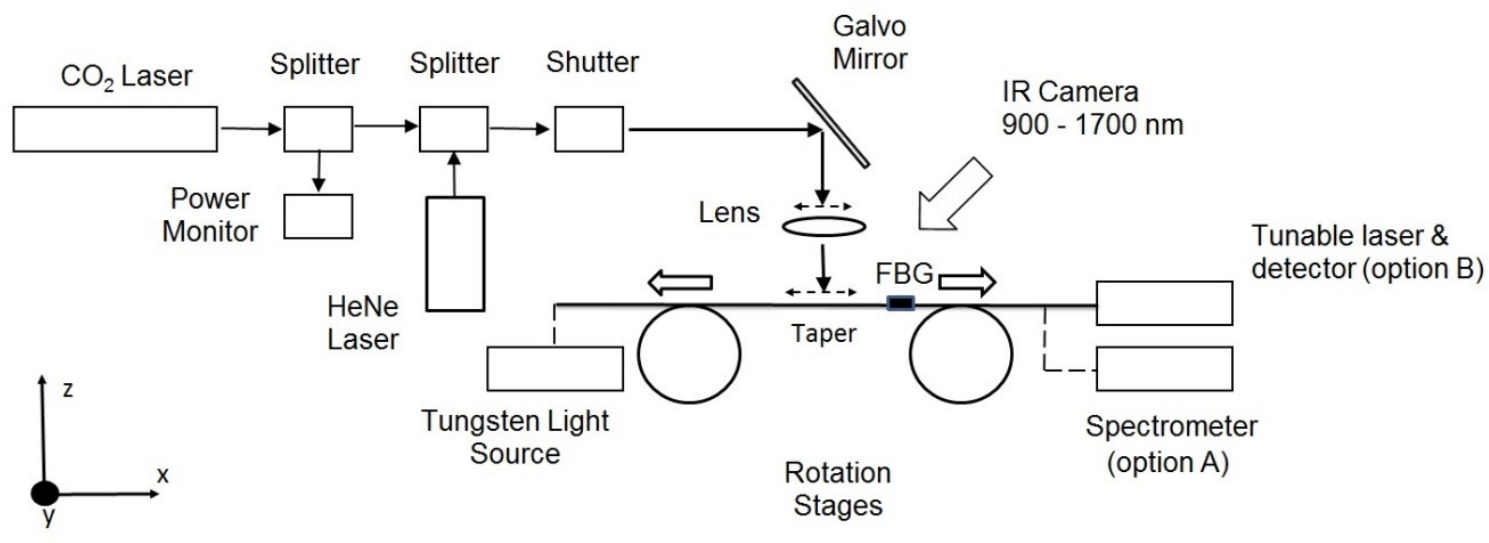

Figure 1. Schematic of experimental system for tapering fibres. 


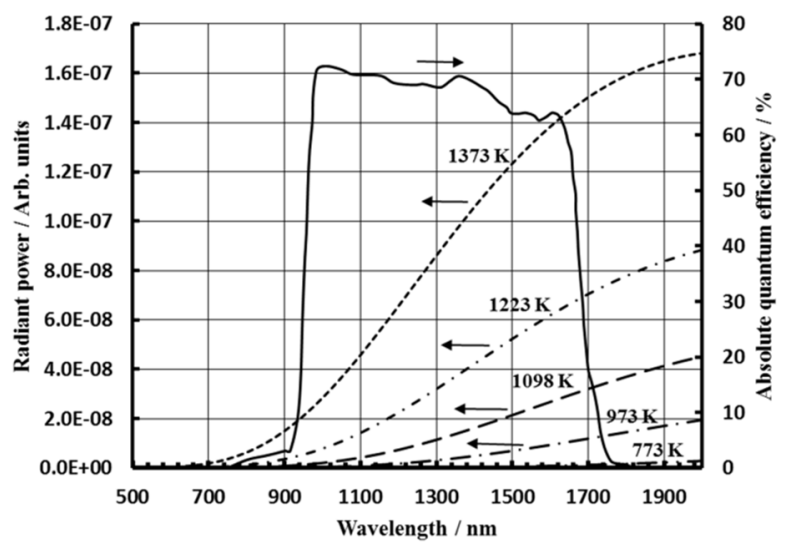

Figure 2. The NIR camera spectral response (solid line) and Planckian black body radiation curves (dashed lines) for a range of temperatures near the fibre melting point of $1183 \mathrm{~K}$.

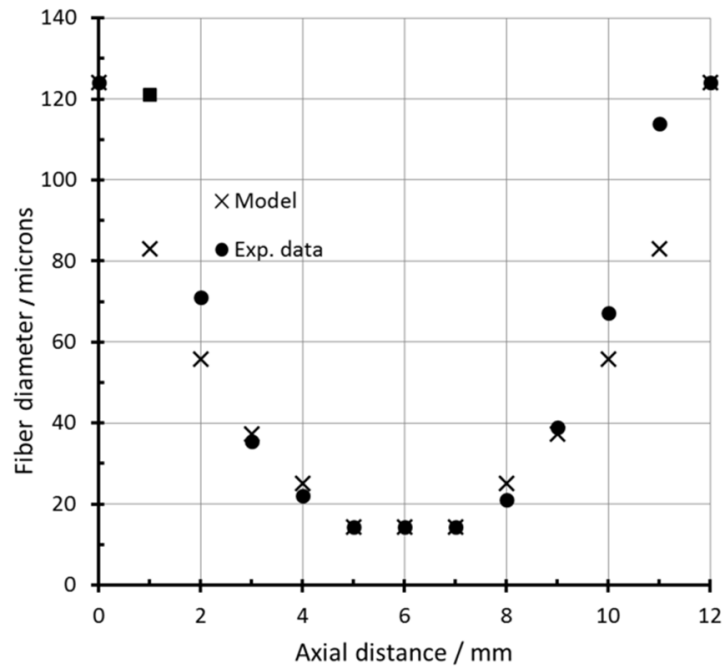

Figure 4. The profile of taper B, fabricated by scanning the laser output over a length of $4.5 \mathrm{~mm}$ and increasing the power from $2 \mathrm{~W}$ to $7 \mathrm{~W}$ as the fibre diameter reduces.

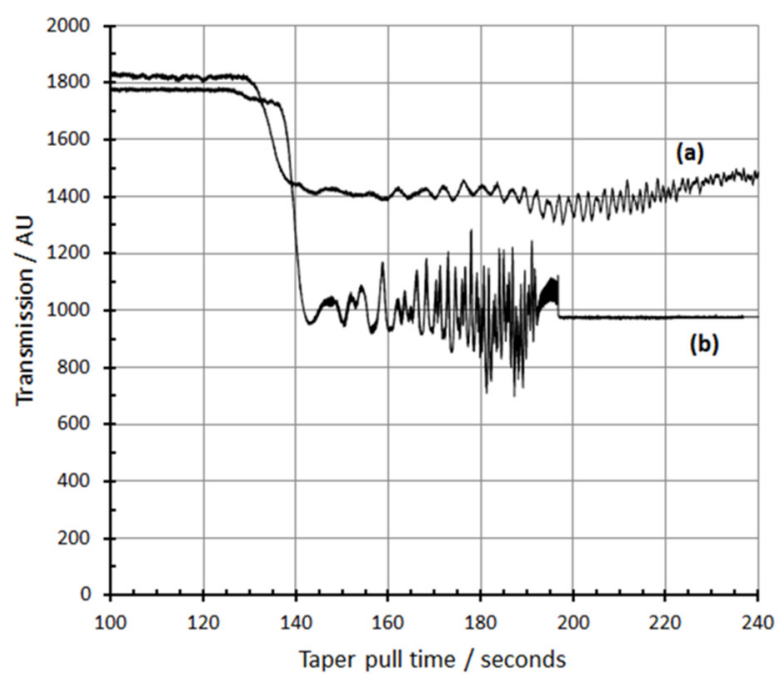

Figure 6 . The transmitted power measured at $800 \mathrm{~nm}$, recorded when fabricating (a) taper A and (b) taper B.

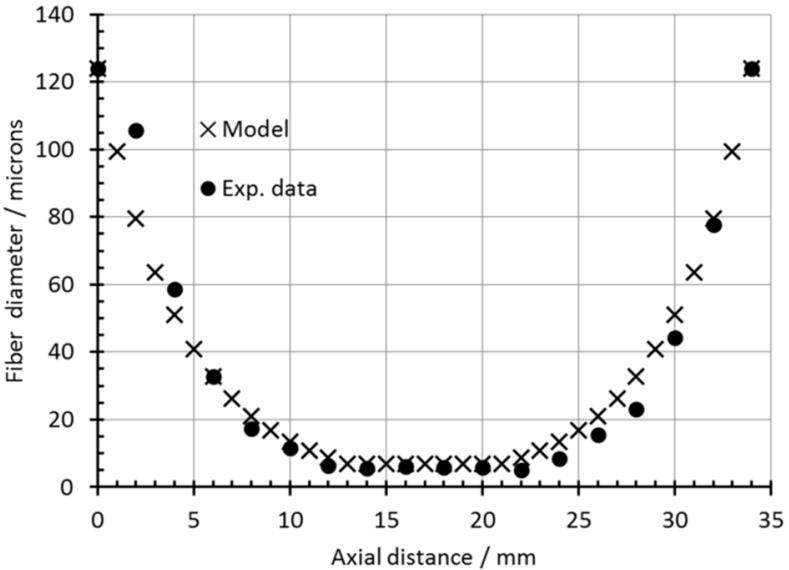

Figure 3. The profile of taper A, fabricated by scanning the laser over a length of $8 \mathrm{~mm}$ and increasing the power from $3 \mathrm{~W}$ to $16 \mathrm{~W}$.

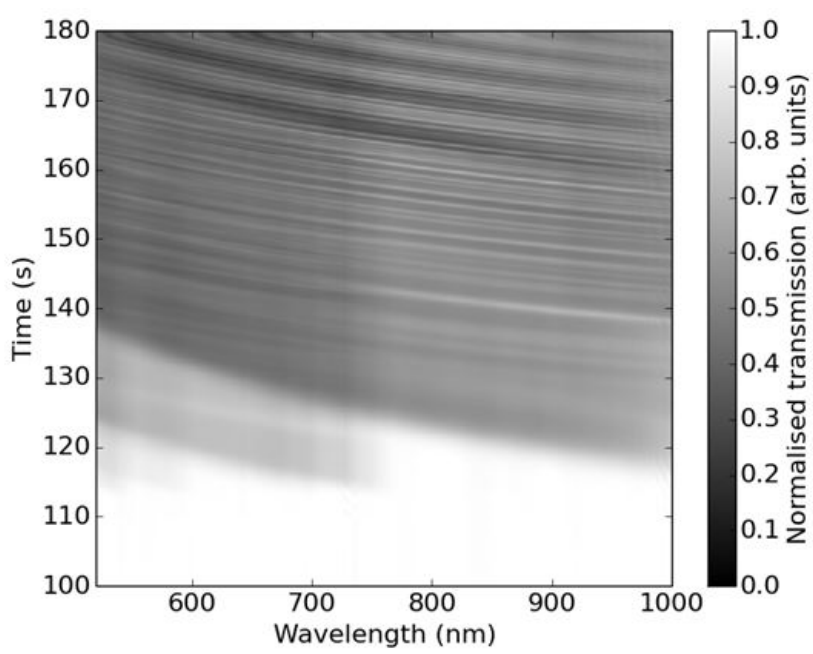

Figure 5. The channelled spectrum during the fabrication of a taper with a similar profile to that of taper B.

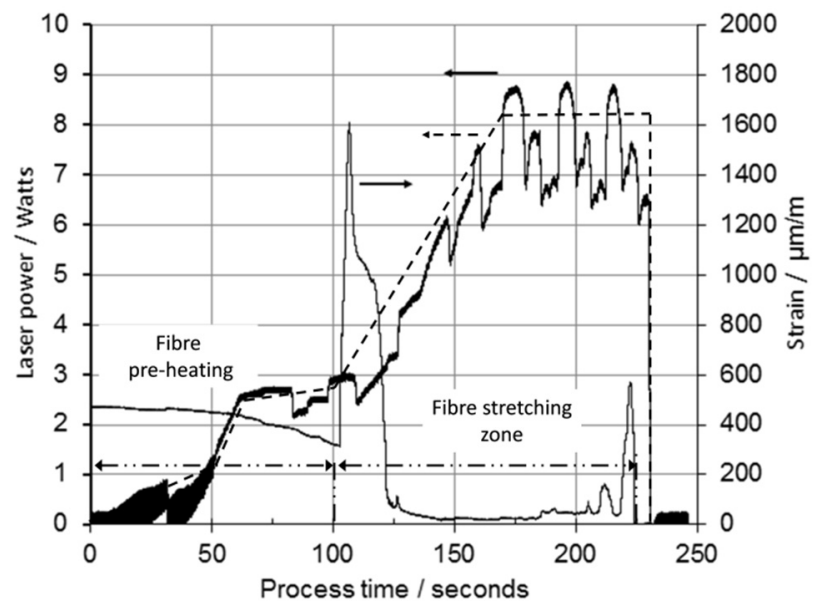

Figure 7. The strain measured by an FBG and laser power during the fabrication of a taper (no pre-heating). The dashed line is the programmed laser power and the dashed/dotted lines defines the fibre heating and stretching zones. 


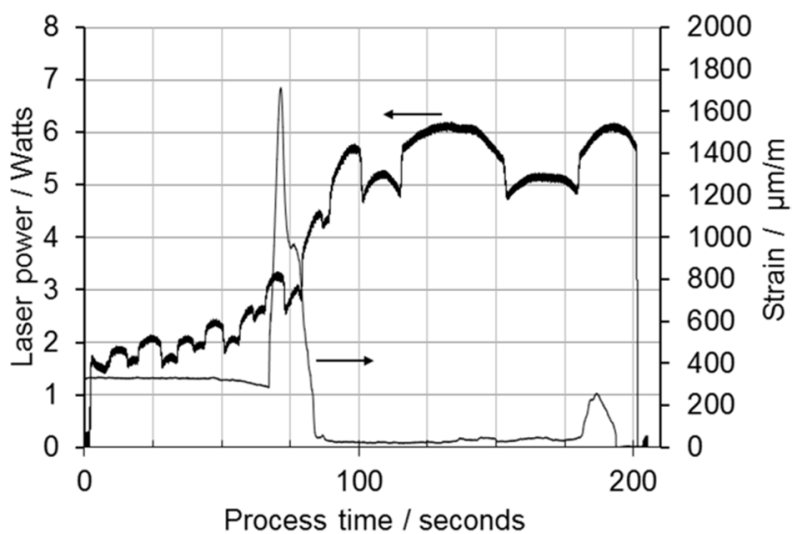

Figure 8. The strain measured by an FBG and laser power during the fabrication of a taper (with pre-heat). 
2015-10-01

\title{
Monitoring techniques for the manufacture of tapered optical fibers
}

\author{
Mullaney, Kevin
}

OSA Publishing

Kevin Mullaney, Ricardo Correia, Stephen E. Staines, Stephen W. James, and Ralph P. Tatam. Monitoring techniques for the manufacture of tapered optical fibers. Environmental Technology, Vol. 54, Issue 28, pp8531-8536 (2015)

http://dx.doi.org/10.1364/AO.54.008531

Downloaded from Cranfield Library Services E-Repository 